\title{
Review
}

\section{Aircraft Ground Handling Operations: A Literary Review}

\author{
Hayat El Asri ${ }^{1}$, Ali Fakhrulddin ${ }^{2}$, Ali Al-Humairi ${ }^{3}$, Najah Al-Mhanna ${ }^{4}$ \\ 1 Coventry University; elasrih@uni.coventry.ac.uk \\ 2,3,4 German University of technology in Oman; ali.fakhrulddin2, ali.alhumari3, \& \\ najah.almhanna@gutech.edu.om4 \\ * Correspondence: elasrih@uni.coventry.ac.uk;
}

\begin{abstract}
Over the past few decades, aircraft Ground Handling Operations (GHO) have been investigated by numerous researchers. Some aspects of GHO have been more focused on than others due to their importance in the GH processes. For instance, GHO performance has been tackled from different perspectives while workers and vehicles allocation suffer from the lack of research in the area. This paper is a literary review of the research that has been conducted in different areas of GHO. It sheds light on GHO performance, resource allocation and scheduling, operations scheduling, vehicle routing, and workers and vehicles allocation.
\end{abstract}

Keywords: Aircraft, Ground Handling, Operations Performance, Resource Allocation, Resource Scheduling, Operations Scheduling, Vehicle Routing, Workers and Vehicles Allocation.

\section{Introduction:}

GHO refer to all the airside activities at airports (Fitouri-Trabelsi et al., 2015). They comprise all the services required by aircraft between landings and take-offs. These services are categorized as follow: ramp, on-ramp, and on-board services. Each of these categories embody several subservices. For instance, ramp services include: marshalling, supervising, parking, and push-back. To ensure proper GH service, several parameters must be taken into account. First and foremost, to eliminate, or at least reduce, delays, GHO performance has to be on point. The proper utilization of resources and the right allocation of workers to vehicles while taking into consideration the routing and the scheduling constraints is vital. Unfortunately, there isn't much literature that tackles GHO issues all together.

This paper is divided into 6 sub-sections. The first section discusses GHO performance. The second section sheds light on vehicle routing in GHO. The third section presents the available literature on GH resource allocation. The fourth section tackles the challenging topic of GH vehicles \& workers' allocation. Operations' Scheduling is the topic covered in the fifth section of this paper. Last but not least, GH resource scheduling is discussed in the last section. A total number of 35 peer-reviewed research papers were studied for this research.

\section{GHO Performance:}

GHO performance embraces several areas such as productivity, efficiency, security, safety, and service quality (ACI, 2012). Monitoring these latter has a direct impact on airports' quality standards (Vreedenburgh, 1999). GHO performance has been tackled by researchers from different angles such as: optimization, operations' costs, and delays. This section discusses the existing literature on GHO.

Nugroho et al. (2012), Bite (2010), Gomez \& Scholz (2009), and Pestana et al. (2010) have all discusses GHO performance from an optimization viewpoint. First, Nugroho et al. (2012) focused 
on specific GHO tasks: baggage handling, passengers handling, and aircraft cleaning, and tried to increase the On Time Performance (OTP) rate. The suggestions made by the researchers consisted of equipment and physical infrastructure; for instance, adding extra desks and information boards to speed up the overall process at airports. The results of the suggested model have been said to improve the performance of ground handling operations; nonetheless, no tests were run to support these claims. Like Nugroho et al. (2012), Bite (2010) has also proposed a way to improve the performance of GHO by introducing new devices that would make communication between vehicles and objects tracking easier. The purpose behind introducing these technologies is to eliminate the delays caused by GH activities and to have more accurate information on any object in real time. The results have shown a decrease in delays in different parts of the airport: GH and passenger movement mainly. To be more specific, the proposed model has shown a decrease of 3 to 5 minutes in baggage searching and a decrease of 1 to 5 minutes in passenger searching. Although this study tackled performance improvement and introduced new technologies, several parameters such as the required time to complete tasks, vehicles routing and workers' allocation were not taken into consideration. Moreover, Gomez \& Scholz (2009) have also suggested ways to improve GHO in reference to the DOC. DOC stands for Direct Operating Costs and refers to all the maintenance and other operations-related expenses. To start, several theories have been proposed. Autonomous pushback was suggested to cut out the unwanted time consumption caused by the current pushback method. Using air-stairs instead of air-bridges was also proposed as a solution to decrease the waiting time of passengers. The DOC calculations were based on the Association of the European Airlines (AEA) method for better assessment of these costs. The results have shown a decrease in the turnaround time. The proposed solutions have a direct positive influence on the DOC of aircraft and can potentially improve the GH environment. Even though this study presents solutions to improve GH performance, the technical aspect of GH was not taken into consideration. Last but not least, Pestana et al. (2010) suggested using an Advanced Surface Movement Guidance and Control Systems (A-SMGCS) approach to model and optimize GH operations. The A-SMGCS system implementation, named A-Guidance, allows efficient communication between the different workers, namely air traffic controller, operations officer, GH manager, and vehicles driver. This system can also integrate already-existing data from the airport; this includes data from the surface monitoring radar, vehicles GPS and others. This system was adopted for the several advantages it offers such as automatic position update 120 times per minute, automatic position broadcast, smaller antennas, automatic transmission, and the ability to use sensors as data sources. It is actually managed at the application server level by the Conflict/Infringement Detection (CID) module. Besides, a Decision Support System (DSS) within the A-Guidance is available to help decision makers. Even though vehicles are taken into account, the drivers, the GH workers, and their availability and skills should be considered.

From a risk management perspective, Fitouri-Trabelsi et al. (2014) proposed a management system based on fuzzy modelling to manage airports' GH operations under risk uncertainty and to improve aircraft GH services at arrival and departure while maintaining low operations' costs. In this research, airlines communicate with the GH personnel through their own stations. For that matter, GH activities were broken down and categorized into three agents to line up with the proposed decentralized management system. The suggested agents are: air traffic controllers, GH managers, and the GH fleet. The proposed solution consisted of having air traffic controllers take care of aircraft schedules while station managers and GH fleet managers communicated together to efficiently schedule and perform all the required tasks. To test the model proposed, aircraft traffic data for 24 hours from Palma de Mallorca Airport on August 1st, 2007 was collected for a case study. The testing phase consisted of applying this heuristic approach to 345 aircraft and comparing the final results. The final results have shown that only 36 aircraft suffered from a maximum delay of 16 minutes. Although this heuristic approach seems promising, a number of important parameters in the turnaround processes, such as the vehicles used by the GH fleet, the workers' schedules, and their availability were not taken into account. 
Furthermore, Samsam (2015) suggested a way to improve the performance of turnaround processes using the Value Operations Methodology (VOM) to reduce the operations' cost from the stakeholders' perspective. A number of improvements were made to the VOM. For example, to rank stakeholders based on their degree of threat and cooperation, a new qualitative method was implemented to replace the old numerical method that was used in the past. Kenya Airways at Jomo Kenyatta Airport was considered as a case study to test and validate the developed model. Data of the GHO performed on Boeing 737-300 for a period of 6 months (January to June 2012) was collected. The results have shown a need for improvement. Therefore, a number of recommendations have been made such as implementing a lean production system for passenger boarding and disembarking and implementing the theory of constraints for cabin grooming.

Niedringhaus (2000), Vidosavljevic \& Tosic (2010), and Wu \& Caves (2007) used software simulations to improve GHO operations. Niedringhaus (2000) proposed a multi-agent based model named Air Carrier Service Evolution (ACSEM) for the airline industry to explore the evolution that the airline industry went through and the improvements that had been made over the years. The simulation was based on data from two airlines, each owning two aircraft in two airports. 1668 aircraft scenarios were used to build the model. The research study, however, does not tackle GH performance directly but rather, the performance of the aviation sector as a whole. Furthermore, Vidosavljevic \& Tosic (2010) proposed a Petri Nets model of turnaround processes to make them more efficient, reliable and time-saving. The proposed model was developed to investigate the allocation strategies, mainly for resources (equipment and staff). The model was built to be dynamic and therefore reachable, boundless, and reversible. To test the model, Belgrade airport was taken as a case study. Four scenarios were investigated. The results have shown that the proposed model could be used and adapted at several levels: pre-tactical, tactical, operational, and strategic. However, the availability of the different resources was not taken into consideration while developing this model. Finally, Wu \& Caves (2007) proposed an analytical model to simulate the performance and efficiency of aircraft turnaround operations. In this model, scheduling buffer time was added into the turnaround time. Data from the European carrier was collected. The results have shown that the use of the buffer has helped reduce the operational cost as it balanced properly between the aircraft utilization and its punctuality. Furthermore, the simulations realized within the scope of this project have shown high turnaround efficiency while maintaining high punctuality. Even though this study proposes a good model that was proven effective, many important parameters such as, but not limited to, workers and vehicles availability were ignored.

Fricke \& Schultz (2009) tackled in their research the delay impacts onto aircraft turnaround performance. In fact, one of the reasons behind flight delays is that some of the GH operations take more than the required time to be completed. The study revealed that there are several factors impacting turnaround reliability such as the tactical aspects, which can be categorized into delayed flight cycles, traffic flow restrictions, ground processes delays, system malfunctions, weather conditions, and other factors. The model presented in this study might allow for optimizing the time buffer as well as finding a balance between minimal buffer times with regard to the delays. However, this paper still does not take into consideration the different tasks required and the workers' allocation, which plays a major role in the overall turnaround performance.

From a managerial standpoint, Alonso (2014) presented a GH management model and a visual interface to provide a complete and concise explanation of how GH works, its management, and the changes that should be made to improve its performance. This work is built on an already proposed model by S. Fitouri Trabelsi (2014), who proposed a hierarchical structure with a GH coordinator responsible for communication between the aircraft and the workers. Furthermore, the concept of Airport Collaborative Decision Making (ACDM) was implemented for performance improvement purposes. The results of the simulation have shown that the developed model is reversible and bounded. Nonetheless, this model was based on a number of assumptions that make the model very general and hardly adaptable to airports. 


\section{Vehicle Routing:}

The vehicle routing problem lies at the heart of distribution management (Cordeau et al., 2007). In aircraft GH operations, the vehicle routing problem was also investigated by many researchers including, but not limited to Di-Yuan et al. (2012), Kuhn \& Loth (2009), and Loth (2011).

Di-Yuan et al. (2012) proposed a greedy randomized adaptive search algorithm to optimize the scheduling of de-icing vehicles in airports. This optimization leads to minimizing the flight delays and achieving the best use of resources. Because of the bottleneck that exists in the Air Transport System (ATS), the efficiency of the entire system depends on individual actors; therefore, optimization is key for efficiency. The structure of this mathematical model for vehicle routing was based on the Vehicle Routing Problem (VRP) and takes into consideration several parameters such as the capacity and the number of operations. A case study based on real-life data for one full day in February 2007 at Stockholm Arlanda Airport was used to test and evaluate the efficiency of the developed algorithm. The proposed algorithms significantly improved the waiting time as well as the delays. It should be noted, however, that although optimization was well done, the workers availability was not taken into account.

Moreover, Kuhn \& Loth (2009) have also proposed a Mixed Integer Linear Program for airport service vehicle scheduling. The timely arrival of vehicles helps ensure the efficient use of resources, i.e. vehicles; therefore, investigating scheduling algorithms is crucial. The timely arrival of vehicles helps ensure the efficient use of resources, i.e. vehicles; therefore, investigating scheduling algorithms is crucial. A case study airport was used for model testing purposes. The results were tested at Hamburg Airport and have shown considerable benefits from the demand-based planning. Furthermore, the delays as well as the vehicles travel distances were reduced by $20 \%$. Although a net improvement on scheduling was attained, workers and other resources were not taken into consideration.

A couple of years later, Loth (2011) proposed a concept and a prototype for vehicle management system in aircraft $\mathrm{GH}$ operations to optimize all GH operations by avoiding delays and reducing the workload and the operations' cost. The proposed vehicle management system has four main elements: the detection and the identification of the different vehicles used in turnaround operations, efficient communication using data link, the provision of traffic data to other vehicles, and finally the availability of scheduling support for the different tasks that need to be performed. Furthermore, a number of simulations were run at Hamburg Airport and at Dallas Fort Worth Airport. The simulations have shown a net amelioration in the delays and a reduction of the vehicles' travel distances. However, the idea behind this research is to improve these results further by installing on-board systems that comprise: a monitoring device, a GPS, a Netbook-PC equipped with a touch display, a power adapter, and a buffer battery on all vehicles. This system was tested in 2 vehicles and 15 luggage cars in the GH area of Hamburg Airport and was deemed very efficient.

\section{GH Resource Allocation:}

GH resource allocation refers to the allocation of the different available resources in the airsides of airport terminals. Several researchers have tackled this domain. This section presents a number of published works within the area.

Justesen (2014) developed a mathematical model for resource allocation in GH operations. A number of problems were faced and addressed. Those problems were broken down into two main categories: airport operations and GHO. Furthermore, the resources dealt with are as follows: check-in counters, stands, gates, and taxi-way routes. Simulation techniques were applied to get a broader idea of the actual problem; which led to the implementation of a greedy heuristic algorithm 
to be developed as a potential solution. After testing the results in Copenhagen \& Frankfurt Airports, a 10\% improvement on the quality and the efficiency of the resources allocation was noticed. Moreover, it was proved that fixing decisions for single flights prior to landing actually results in a major quality loss; therefore, looking at all flights arriving within a 45-minute interval is necessary to allocate resources efficiently. Although the results presented in this research are convincing, the workers and the vehicles schedules and availability were not taken into consideration.

Moreover, Dohn \& Kolind (2009) have also developed a model for the optimization of workforce allocation in GHO by maximizing the tasks number while using resources as efficiently as possible. The main problem addressed here is the proper assignment of the different worker teams to the different tasks to be performed while taking some restrictions into account. The results have shown optimal solutions: enforcement of synchronized execution of tasks as well as an efficient use of resources. Although this research study takes both workers and tasks into consideration, the vehicles to be used are ignored. It should be noted that vehicles scheduling may impact the correctness of this model.

Besides, Kabongo (2015) has also proposed a multi-agent based model for GH management at airports to increase the efficiency as well as the effectiveness of aircraft handling and turnaround time in a reduced time. Allocating resources for $\mathrm{GHO}$ was looked at from a mathematical perspective without taking any uncertainty or risk into consideration. To test the model, Brasilia Airport was used as a case study. The results of this research were not discussed in the paper; the future works section therein states that the results will be discussed in a later paper.

Fitouri-Trabelsi et al. (2015) suggested a hierarchical structure based on a heuristic algorithm for airports GH management to make GH resources available in a way that all aircraft would be served in the minimum possible delay. For that, two contrasting scenarios had to be considered: normal versus disturbed conditions. A multi-agent model that comprises three agents, namely the aircraft, air traffic controllers, and the GH coordinators was developed. Each of these agents has specific tasks to fulfil and must keep the other agents updated at all times. To test and validate the proposed model, Palma de Mallorca airport was taken as a case study. Real-life data from this airport on 01 August 2007 for a total period of 24 hours was used. During this period, 690 aircraft landed and took off (all airport terminals combined). The results of the proposed model have shown that of all aircraft, only 14 will experience a delay of 14 minutes maximum. Yet, although the proposed model's tests came out conclusive, considering that all GH operations need 5 minutes to complete was not realistic. Also, the workers and vehicles were not taken into account in this research.

Kelemen (2005) proposed a resource management system for aircraft GH operations in order to develop a structured resource management system for efficient management of both mobile and immobile resources. In this case, immobile resources represent immobile equipment such as desks and check-in counters, while the mobile resources refer to the airport staff, vehicles, and all the other mobile equipment. The proposed system has five different modules: check-in allocation, gate allocation, baggage allocation, staff allocation and equipment allocation. This system will display Gantt charts for each of these modules. Nonetheless, this system does not take into account the workers and equipment allocation together.

Marintseva et al. (2015) proposed a resource allocation improvement for aircraft GHO. Because GHO are performed on two different sites: landside and airside, this paper introduced two subdivisions: aircraft GH and terminal handling. A mathematics-based algorithm was proposed to reduce the total turnaround time while optimizing the number of staff. To test and validate the results of this study, Kyiv Airport was taken as a case study. Although this paper provides a model 
to optimize the turnaround time and staff, several conditions, such as the vehicles and workers' availability, were not taken into consideration.

\section{GH Vehicles \& Workers Allocation:}

Aircraft GH vehicles and workers' allocation refers to the optimization of vehicles to workers. This research area has not been investigated a lot. The papers below present different views of vehicles and workers' allocation.

Jorg Herbers (2005) tackled in his research the optimization problems that exist in the planning stages of GHO. For that matter, two algorithms were used mainly for task level shift planning (related to vehicle routing) and shift scheduling models. Moreover, another algorithm was developed for complex cyclic rostering. This latter is built upon an already existing model for solving both shift scheduling and cyclic rostering concurrently. The experiments carried out proved the efficiency of the proposed model. In fact, compared to a heuristic algorithm, this model has shown a decrease of $22.7 \%$ in shift costs while keeping a moderate solution times. Although the proposed model was proven effective, it only focused on the planning of tasks and does not take into account the availability of workers, their schedules, and the availability of the vehicles to be used.

Chung et al. (2010) proposed a multi-agent based model for airport service planning. To increase airports' competitiveness and reduce the operations' cost at the same time, optimizing maintenance vehicles allocation was put forward as a potential solution to the problem. A model containing five agents was built: aircrafts agent, cleaning vehicles agent, water vehicles agent, tractors agent, and the decision maker agent. In this model, communication between the aircraft agent and the other vehicle agents must go through the agent decision maker, who checks the availability of the different vehicles depending on the current need. Hong Kong International Airport, China was taken as a case study in this paper to test the efficiency of the proposed multi-agent based model. The implementation of this model showed a clear optimization of the vehicles being used and prevented delays. Although this model had taken into consideration different agents and established good communication between them, it did not take into consideration the different types of workers that work in GHO. Workers allocation was only based on the assumption that different workers are embedded within different vehicles.

Fink et al. (2016) proposed column generation for vehicle routing problems while taking into account multiple synchronization constraints. The research focused on operational GH planning and modelling. For that matter, the Vehicle Routing Problem with Workers and Vehicles Synchronization (AVRPWVS) mathematical model has been suggested. This AVRPWVS's main purpose is to deal with both workers and vehicles in GH and to minimize the delays as well as the vehicle routing costs. Five types of synchronizations are considered in this AVRPWVS problem: resource synchronization, operation synchronization, movement synchronization, task synchronization, and load synchronization. The purpose of all of these is to synchronize all elements that are related to vehicle routing. Moreover, two algorithms have been used for the model: a labelling algorithm and an extended vector method algorithm. Also, a branch-and-price heuristic algorithm was developed for the purpose of fixing the modified routes. The results have shown that the Branch and Price Heuristic (BAPH) and the MILP have almost the same gap and run for almost the same amount of time. This model, however, has not been tested on a real-life scenario. Therefore, the results presented may not be accurate.

\section{GHO scheduling:}

Concerning GHO scheduling, Guimarans et al. (2015) proposed an optimization approach to cut out the delays, minimize the operations' waiting time, and optimize the total turnaround time. 
Constraint Programming (CP) was used as a technique to implement the model. Furthermore, Guimarans et al. (2015) took a number of parameters into consideration such as aircraft arrival and departure times and the different turnaround operations' pre-requisites. The results of this research have shown that different approaches could be adopted for the operations scheduling; however, no mathematical results have been presented. Even though this paper takes both operations scheduling and vehicle routing into account, it does not mention the workers, which are an important part of every operation.

Furthermore, S.P. Astorga (2014) suggested an optimization approach similar to what Guimarans et al. (2015) proposed. The main objectives were to mainly to minimize the delays. S.P. Astorga (2014) has also opted for Constraint Programming (CP) as a technique and a number of other algorithms such as a Variable Neighborhood Descent (VND), Large Neighborhood Search (LNS), and an Insertion Heuristics Method (IHM) were used. Like Guimarans et al. (2015), no mathematical results were presented. Nevertheless, the system was said to allow proper scheduling of operations and was tested in 2 airports: Palma de Mallorca airport and Barcelona airport. The results have shown that the proposed model allows proper scheduling of GH vehicles within the given time frame. The workers, however, were not considered in this research.

From another dimension, Bevilacqua et al. (2014) proposed another approach for ground handling operations scheduling. The system consists of applying Business Process Re-Engineering (BPR) to analyze the impact of the GHO growth. Unlike Guimarans et al. (2015) and S.P. Astorga (2014), Bevilacqua et al. (2014) used different tools and techniques such as the Delphi method, IDEF3, and discrete event simulation. Moreover, the focus was mainly on the "as-is" scenarios and ways to improve and optimize it in order to achieve the "should-be" scenario. Likewise, a number of suggestions have been made in order to optimize the operations scheduling; for instance, increasing the staff member was proposed as a potential solution. Nonetheless, this simulation did not account for the workers' skills and availability as well as vehicles scheduling.

From a computer simulation perspective, Voulgarellis et al. (2005) have also developed a simulation model to optimize GHO scheduling. Unlike Bevilacqua et al. (2014), Voulgarellis et al. (2005) have opted for MATLAB as a programming language and SLAGOM as simulation language. The focus was on some specific parts of ground handling, namely: runway, taxiway, and stands. The results have shown an improvement in the operations planning. Nonetheless, some important factors, for instance, the ground handler workers, the different vehicles used for the different tasks, the specialized vehicles, and the availability of both workers and vehicles, were not taken into account.

Following the same approach, Ioannidis et al. (2005) have also proposed a Matlab based simulation for ground handling operations scheduling. This paper, however, was based on a number of assumptions. For example, airports were considered to only have one runway and one taxiway. Besides, a number of algorithms were embedded within a mathematical model were developed to achieve the main research objective. Last but not least, the system was proven flexible, dynamic, and compatible when tested. Nonetheless, although this paper tackles the operations scheduling issue and tries to improve it, it does not take into account all the elements that are involved in all the GH operations such as the workers, the vehicles, and the availability of both of these.

\section{GH Resource Scheduling:}

GH resource scheduling refers to the planning and the scheduling of the different available resources in airports' airside. Several researchers have investigated the existing issues in this domain.

Mao et al. (2007) proposed a multi-project scheduling of airport GH under uncertainty. The GH operations and services are, most of the time, considered autonomous parties, which causes delays 
and disturbances. As a solution to this problem, Mao et al. proposed a heterogeneous multi-agent framework. This latter has two types of agents: the aircraft and the GH operations; both agents are independent to allow them to consider individual benefits while making scheduling decisions. Furthermore, online scheduling is used to eliminate the risk of rescheduling under uncertainty, while a market-based mechanism for the project activities is used mainly to assign turnaround operations timeslots. Taking into consideration the dynamic environment of airports, the cancellation of operations and/or their rescheduling is common. To cope up with this issue, the insertion of slack time between different activities was considered as a solution. An airport specialized MPSP (Multi-Project Scheduling Problem) was used as a case study to test the results of the suggested solution. The final results have shown that the proposed model responds correctly under uncertainty and does not present any flaws. Aircraft arrivals scheduling under uncertainty was completely eliminated thanks to the proposed model. Also, disruptions triggered by GH incidents were reduced considerably. Nonetheless, some incidents were noted due to the inefficiency of resources. Although the scheduling issue was solved, some other parameters, mainly the workers and vehicles availability, should have been taken into consideration in order to make this system more efficient.

Clausen (2011) investigated the problem of airport ground staff scheduling to have a closer look at workforce planning as well as task scheduling and ways to improve them, and thus proposed a planning model. This latter was divided into three distinct categories, each with a specific objective: strategic planning, tactical planning, and real-time planning. Because airport workforce scheduling efficiency depends on a number of factors such as the start and the end of an operation, combining dynamic optimization with distributive management was necessary in order to be able to cope with the different scenarios that may occur. Moreover, mathematical modelling was used to simulate the proposed solution. The results have shown improvements on the airport scheduling. Nevertheless, several parameters were not taken into consideration. For instance, staff allocation should take into consideration the different tasks assigned to each worker.

Norin et al. (2012) presented a model for logistics planning using Stockholm Arlanda Airport as a case study. Prior to implementing the model, optimization techniques have been integrated into the simulation model to calculate the airport performance indicators. because of the already established schedules at Stockholm Arlanda Airport, some tasks can be performed at the same time, which has to be taken into account when building the model. The main aim of this paper is to optimize one particular process: de-icing. For this matter, a GRASP heuristic algorithm was applied. To test these results, four scenarios, when de-icing is not considered versus when it is considered, were created. The simulation ran for 24 hours and included 515 flights at Stockholm Arlanda Airport in 2008. 50 replications were needed for each of the four proposed scenarios. The different simulations helped the researchers choose the best scenario to implement from an optimization perspective. This paper, however, only focuses on one task in the GH operations and does not take into consideration the availability of the worker(s) who will be driving the de-icing vehicle.

Ansola et al. (2014) proposed a distributed agent-based model to improve the resources scheduling in aircraft GH operations. Because airport operators have started incorporating what is called a "common use of resources", computer software and new algorithms and techniques need to be put in place to mainly monitor the utilization of resources. A "common use of resources" refers to sharing the available resources in order to increase the operations' efficiency and passengers' processing options. For that matter, this research aims at providing a decision-making framework to provide short-run schedules for GH operations. Artificial intelligence, and more specifically agent based systems, are used. This proposed system is easy to integrate with other environments such as Enterprise Resource Planning (ERP) and Customer Relationship Management (CRM) systems that may be used within airports. In addition, three constraints were taken into account: the utilization of resources, the expected duration, the coordination of different elements. After testing and validating the proposed model in labs, Ciudad Real Central Airport, Spain, was taken as a case 
study. The results have shown a clear reduction in the utilization of resources. This paper takes into consideration all the tasks carried out in the GH operations. However, it does not take the workers' skills and availability into account.

Kuster \& Jannach (2005) proposed a way for handling airport GH processes based on the Resource Constraint Project Scheduling (RCPSP) framework. This framework is said to be efficient and appropriate for the scheduling and re-scheduling problems faced in turnaround operations. Because of the lack of support in the RCPSP version, an extended new version (x-RCPSP) was proposed within the scope of this research. Turnaround processes with disruption were examined, and a number of suggestions were made to reduce the delays. The implementation, testing, and validation of these algorithms have been proved efficient in real-time decision support in disruption management. Although this paper considers many factors that are related to GH operations scheduling, workers were not taken into account.

\section{References}

Fitouri-Trabelsi, S., Mora-Camino, F., Nunes-Cosenza, C. and Weigang, L. (2015). Integrated Decision Making for Ground Handling Management. Global Journal of Science Frontier Research: F Mathematics and Decision Sciences, [online] 15(1). Available at: https://hal-enac.archives-ouvertes.fr/hal-01117103/document [Accessed Mar. 2017].

ACI (2012). Annual Report 2012. [online] Aci-bd.com. Available at: http://www.acibd.com/finance/aci/annual/ACI\%20Annual\%20report\%202012\%20pdf\%20file.pdf [Accessed 5 Sep. 2018].

Vreedenburgh, M. (1999). Airport Operational Efficiency. [online] International Civil Aviation Organization (ICAO). Available at: https://www.icao.int/Meetings/AMC/MA/1999/aps/05_vreedenburgh.pdf [Accessed 5 Apr. 2017].

Nugroho, I., Riastuti, U. and Iridiastadi, H. (2012). Procedia - Social and Behavioral Sciences. Performance Improvement Suggestions for Ground Handling using Lean Solutions Approach. 65, pp.462-467.

BITE, K. (2010). IMPROVING ON AIRPORT'S GROUND HANDLING. [online] Available at:

http://www.wctrs.leeds.ac.uk/wp/wp-content/uploads/abstracts/lisbon/general/02868.pdf [Accessed Mar. 2017].

Gomez, F. and Scholz, D. (2009). Improvements to Ground Handling Operations and Their Benefits to Direct Operating Costs. Deutscher Luft- und Raumfahrtkongress, [online] pp.1-11. Available at: http://www.fzt.haw-

hamburg.de/pers/Scholz/ALOHA/ALOHA_PUB_DLRK_09-09-08.pdf [Accessed Mar. 2017].

Pestana, G., Rocha da Silva, T. and Reis, P. (2011). IEEE. Handling Airport Ground Operations using an A-SMGCS Approach. [online] pp.1-15. Available at: http://ieeexplore.ieee.org/document/5747371/metrics?part=1 [Accessed 11 Apr. 2017].

Fitouri-Trabelsi, S., Cosenza, C., El Moudani, W. and Mora-Camino, F. (2014). Managing uncertainty at airports ground handling. [online] Available at: http://www.stac.aviation-civile.gouv.fr/manifestation/tra2014/presentations/4AUN2014_Managing_Uncertainlye.pdf [Accessed Mar. 2017].

Niedringhaus, W. (2000). An agent-based model of the airline industry. [online] Available at: https://www.caasd.org/library/papers/ACSEM.pdf [Accessed Mar. 2017].

Vidosavljević, A. and Tošić, V. (2010). Modeling of Turnaround Process Using Petri Nets. [online] Available at: http://MODELING OF TURNAROUND PROCESS USING PETRI NETS [Accessed Mar. 2017].

Wu, C. and Caves, R. (2007). Modelling and optimization of aircraft turnaround time at an airport. [online] Available at: http://www.aviation.unsw.edu.au/downloads/papersWu/1_TP\&T_V27N1_2004_2.pdf [Accessed 19 Mar. 2017].

Fricke, H. and Schultz, M. (2009). Delay Impacts onto Turnaround Performance: Optimal Time Buffering for Minimizing Delay Propagation. [online] Available at: http:// www.atmseminar.org/seminarContent/seminar8/papers/p_153_AO.pdf [Accessed Mar. 2017]. 
Alonso, B. (2017). Ground handling management modelling and visual interface conceptual design [online]. Available at: http://bibing.us.es/proyectos/abreproy/60213/fichero/PFC+Beatriz+Arias+Alonso+-

+Ground+Handling+(ingl\%C3\%A9s).pdf [Accessed Mar. 2017].

Cordeau, J. (2007). Unal Edu. Vehicle Routing. [online] Available at:

http://dis.unal.edu.co/ gjhernandezp/TOS/ROUTING/VRP1.pdf [Accessed 4 Aug. 2017].

Kuhn, K. and Loth, S. (2009). Airport Service Vehicle Scheduling. [online] Available at: http://www.atmseminar.org/seminarContent/seminar8/papers/p_007_AO.pdf [Accessed Mar. 2017].

Loth, S. (2017). Concept and prototype of a ground handling vehicle management system. [online] Available at: http://www.atmseminar.org/seminarContent/seminar9/presentations/66-Loth-PresentationPDF-6-3-11.pdf [Accessed 17 Apr. 2017].

Justesen, T., Dohn, A. and Larsen, J. (2014). Allocation of Ground Handling Resources at Copenhagen Airport. [online] Available at: http://orbit.dtu.dk/files/103714768/Tor_Fog_Justesen_Afhandling.pdf [Accessed 1 Mar. 2017].

Kabongo, P. (2015). A Multi Agent Based Model for Ground Handling Management at Airports. [online] Available at: http://www.translab.unb.br/images/slides/14-ground-handling-management.pdf [Accessed Mar. 2017].

Dohn, A. and Kolind, E. (2017). Optimizing Manpower Allocation for Ground Handling Tasks in Airports using Column Generation. [online] Available at:

http://www2.imm.dtu.dk/pubdb/views/edoc_download.php/5712/pdf/imm5712.pdf [Accessed Mar. 2017].

Fitouri-Trabelsi, S., Mora-Camino, F., Nunes-Cosenza, C. and Weigang, L. (2015). Global Journal of Science Frontier Research: F Mathematics and Decision Sciences. Integrated Decision Making for Ground Handling Management. [online] 15(1). Available at: https://hal-enac.archives-ouvertes.fr/hal-01117103/document [Accessed Mar. 2017].

Marintseva, K., Yun, G. and Kachur, S. (2015). Taylor \& Francis Group. Resource allocation improvement in the tasks of airport ground handling operations. [online] 19, pp.7-13. Available at: http://dx.doi.org/10.3846/16487788.2015.1015291 [Accessed 15 Apr. 2017].

Chung, N., Ip, W., Cho, V. and Ho, G. (2010). A Multi Agent Based Model for Airport Service Planning [online]. Available at: http://hrcak.srce.hr/file/98301 [Accessed Mar. 2017].

Fink, M., Desaulniers, G., Soumis, F., Frey, M., Kiermaier, F. and Kolisch, R. (2016). Gerad.Column generation for vehicle routing problems with multiple synchronization constraints. [online] Available at: https://www.gerad.ca/fr/papers/G-2016-63 [Accessed 17 Apr. 2017].

Guimarans, D., Padrón, S., Ramos, J. and Fitouri-Trabelsi, S. (2015). Lecture Notes in Management Science. Scheduling ground-handling services: a bi-objective approach. [online] 7, pp.27-33. Available at:

http://orlabanalytics.ca/lnms/archive/v7/lnmsv7p27.pdf [Accessed Mar. 2017].

Astorga, P. (2017). a multi objective optimization approach to the ground handling management problem. [online] Available at:

http://www.tdx.cat/bitstream/handle/10803/284947/spa1de1.pdf;jsessionid=2A7ED87A82B9394CD0ED4F8956D3CF9E?seque nce=1 [Accessed Mar. 2017].

Bevilacqua, M., Ciarapica, F., Mazzuto, G. and Paciarotti, C. (2014). The impact of business growth in the operation activities: a case study of aircraft ground handling operations. Production Planning \& Control, 26(7), pp.564-587.

Voulgarellis, P., Christodoulou, M. and Boutalis, Y. (2005). A MATLAB Based Simulation Language for Aircraft Ground Handling Operations at Hub Airports (SLAGOM). Mediterranean Conference on Control and Automation, [online] pp.334-339. Available at: http://ieeexplore.ieee.org/document/1467037/ [Accessed 13 Apr. 2017]. 
Ioannidis, G., Christodoulou, M. and Boutalis, Y. (2005). Mediterranean Conference on Control and Automation. A SIMULINK Based Simulation Language for Aircraft Simulation of Ground Handling Operations at Hub Airports (SSLA). [online] pp.340-345. Available at: http://locate.coventry.ac.uk/primo_library/ [Accessed 10 Apr. 2017].

Mao, X., Roos, N. and Salden, A. (2007). Stable Multi-project Scheduling of Airport Ground Handling Services by Heterogeneous Agents. [online] Available at: https://dke.maastrichtuniversity.nl/nico.roos/wpcontent/uploads/2016/01/MRS09AAMAS.pdf [Accessed Mar. 2017].

Clausen, T. (2017). Airport Ground Staff Scheduling. [online] Orbit.dtu.dk. Available at: http://orbit.dtu.dk/fedora/objects/orbit:86371/datastreams/file_5682298/content [Accessed Mar. 2017].

Norin, A., Yuan, D., Andersson Granberg, T. and Värbrand, P. (2012). Scheduling De-icing Vehicles within Airport Logistics: A Heuristic Algorithm and Performance Evaluation. [online] Available at: http://citeseerx.ist.psu.edu/viewdoc/summary?doi=10.1.1.650.7973 [Accessed Mar. 2017].

Garcia Ansola, P., Garcia Higuera, A., Otamendi, F. and de las Morenas, J. (2014). Agent-Based Distributed Control for Improving Complex Resource Scheduling: Application to Airport Ground Handling Operations. IEEE Systems Journal, [online] 8(4), pp.1145-1157. Available at: http://ieeexplore.ieee.org/document/6575111/ [Accessed 13 Apr. 2017].

Kuster, J. and Jannach, D. (2005). Handling Airport Ground Processes based on Resource-Constrained Project Scheduling. [online] Available at: http://ls13-www.cs.tu-dortmund.de/

homepage/publications/jannach/Conference_IEA_2006_CDM.pdf [Accessed 12 Apr. 2017]. 